questions limit the significance of the findings: 1) Should the children be accepted as "healthy"? They were referred for EEG because of minor head trauma, and the sequelae of mild traumatic brain injuries are controversial and sometimes serious (Lee LK. Pediatr Emerg Care 2007;23:580-583); Hamilton M et al. Incidence of delayed intracranial hemorrhage in children after uncomplicated minor head injuries. Pediatrics July 2010;126:e33-e36); 2) A proportion of the patients with epileptiform EEG discharges developed afebrile seizures and some had seizure recurrences and epilepsy. These should have been excluded from the data; 3 ) Of 7 previous published reports of EEG epileptiform discharges in nonepileptic children with ADHD the authors selected only 2 , both with the lowest prevalence of abnormalities; 5 previous reports with significantly higher prevalences were omitted from their discussion.

In a recent study of 612 children evaluated for symptoms of ADHD complicated by episodic altered awareness, the frequency of epileptiform abnormalities in the sleepdeprived EEG was $25.7 \%$ and similar to the mean frequency for 7 published studies (Millichap JJ, Stack CV, Millichap JG. J Child Neurol 2010; in press). Our findings and previous reports are not in agreement with the current German study conclusions. The weight of evidence favors an increased prevalence of epileptiform EEGs in non-epileptic children with ADHD.

When stimulant medication is prescribed in ADHD children with abnormal EEG, a conservative dose schedule may be advisable. In a study of children with ADHD complicated by EEG rolandic spikes, seizures occurred in $16.7 \%$ following methylphenidate compared with $0.6 \%$ in a group of ADHD children with normal EEGs. (Hemmer SA et al. Pediatr Neurol 2001;24:99-102). The safety of high-dose stimulants in children with ADHD and epileptiform EEGs untreated with antiepileptic medication is unclear. An EEG may allow the physician and parent to make an informed decision regarding risks and benefits of high-dose stimulant versus nonstimulant selection and immediate-release MPH vs extended-release formulations.

OROS-MPH (Concerta) treatment of ADHD with epilepsy. A current report of a randomized control trial of OROS-methylphenidate, $18-54 \mathrm{mg}$ daily for weekly periods, in 33 patients, 6-18 years of age, taking antiepileptic drugs, found an increased daily risk of seizures with increasing dose of OROS-MPH, an extended-release medication. Potential safety concerns require further study. (Gonzalez-Heydrich J, Biederman J et al. Epilepsy Behav July 2010;18:229-237).

\title{
ALDH7A1 DEFICIENCY AND PYRIDOXINE-DEPENDENT EPILEPSY
}

Researchers at University College and Great Ormond Street Hospital for Children, London, and other centers in the UK and Europe investigated the genotypic and phenotypic spectrum of pyridoxine-dependent epilepsy (PDE) by measurement of urinary alpha-aminoadipic semialdehyde (a-AASA) concentration and mutational analysis of the ALDH7A1 gene that encodes antiquitin. Twenty-one new patients with elevated AASA and 37 individuals from 30 families with mutations in ALDH7A1 were identified; 17 of these were novel and all others were published previously. The clinical spectrum of antiquitin deficiency and pyridoxine-dependent epilepsy includes ventriculomegaly, 
abnormal fetal movements, multisystem neonatal disorder, and seizures and autistic features after the first year of life. Presenting features included metabolic acidosis, respiratory distress, hypotonia, food intolerance, prematurity and fetal distress. Electrolyte derangements sometimes associated may include hypocalcemia and hypomagnesaemia, and endocrine disturbances include hypothyroidism and diabetes insipidus. Neurodevelopmental outcome is impaired in the majority of cases. Diagnosis of pyridoxin dependent epilepsy is sometimes challenging because seizures are responsive to anticonvulsant drugs in $38 \%$ cases, response to pyridoxin may not be instant and obvious in $14 \%$, and structural brain abnormalities sufficient to explain the epilepsy may coexist.

Seizure types were clonic (91\%), myoclonic jerks $(62 \%)$ and tonic $(44 \%)$. EEG abnormalities included burst suppression (21\%) and hypsarrythmia (5\%). Some movement disorders were dystonic and not associated with EEG changes. MRI abnormalities inciuded cortical dysplasia, corpus callosal agenesis, and hydroccphalus, requiring v-p shunt. Biochemical and DNA tests for antiquitin deficiency and a clinical trial of pyridoxine are recommended in infants and children with epilepsy and a variety of clinical features. A clinical trial of pyridoxine should be continued for a minimum of 72 hours, with careful clinical and EEG monitoring. (Mills PB, Footitt EJ, Mills KA, et al. Genotypic and phenotypic spectrum of pyridoxine-dependent epilepsy (ALDH7A1 deficiency). Brain July 2010;133:2148-2159). (Respond: Prof PT Clayton, UCL Institute of Child Health, 30 Guilford Street, London WC1N 1EH, UK. E-Mail: p.clayton@ich.ucl.ac.uk).

COMMENT. In neonates and infants with intractable seizures, measurement of urinary alpha-aminoadipic semialdehyde and DNA tests for antiquitin deficiency should be performed to exclude pyridoxin-dependent epilepsy (PDE), regardless of an associated electrolyte disorder or structural abnormality on brain MRI. Diagnosis may be challenging because the seizures are sometimes partially controlled by anticonvulsant drugs, and the response to pyridoxine may be delayed. In classical cases of PDE, seizures occur in the first month, often within hours of birth. They are resistant to antiepileptic drugs and are controlled within an hour by $50-100 \mathrm{mg}$ pyridoxin given intravenously. A maintenance dose of $5-10 \mathrm{mg} / \mathrm{kg} /$ day of oral pyridoxin is usually sufficient to control the seizures.

\section{DIAGNOSTIC UTILITY OF THE ICTAL CRY}

Consecutive video-EEGs of 20 adult patients with generalized tonic-clonic (GTC) seizures and 20 with psychogenic nonepileptic seizures (PNES) were reviewed from the archives at University of South Florida, Tampa General Hospital, and the audio components of the recordings were analyzed and compared for all vocalizations. The ictal cry was defined as a prolonged tonic expiratory laryngeal vocalization, or a deep guttural clonic vocalization. The typical laryngeal sound had high sensitivity $(85 \%)$ and specificity $(100 \%)$ for epileptic GTC seizures and was not heard in any of the psychogenic cases. PNES utterances were weeping, moaning, and coughing. The ictal cry was strongly associated with epileptic GTC and warrants inquiry when taking the history from witnesses of a patient's seizure. 